\title{
Electronic Dispersion Precompensation of Direct-Detected NRZ using Analog Filtering
}

\author{
M. Verplaetse, L. Breyne, J. Van Kerrebrouck, P. Ossieur and G. Torfs
}

\begin{abstract}
We demonstrate (in real-time) electrical dispersion compensation in direct detection links using analog transmit side filtering techniques. By this means, we extend the fiber reach using a low complexity solution while avoiding digital preprocessing and digital-to-analog converters (DACs) which are commonly used nowadays. Modulation is done using an IQ MachZehnder modulator (MZM) which allows straightforward compensation of the complex impulse response caused by chromatic dispersion in the fiber. A SiGe BiCMOS 5-tap analog complex finite impulse response (FIR) filter chip and/or a delay between both driving signals of the MZMs is proposed for the filter implementation. Several link experiments are conducted in C-band where transmission up to $60 \mathrm{~km}$ of standard single-mode fiber (SSMF) of direct detected $28 \mathrm{~Gb} / \mathrm{s} \mathrm{NRZ/OOK}$ is demonstrated. The presented technique can be used in applications where low power consumption is critical.
\end{abstract}

Index Terms-Direct Detection, Equalizers, Chromatic Dispersion, Analog Integrated Circuits, EDC, Electronic predistortion

\section{INTRODUCTION}

D IRECT detection (DD) provides a low-cost, low power consumption and simple solution for fiber-optic links, and is the preferred solution for intra data center, inter data center and intermediate reach metro links. However, the link reach and data rate are limited by the chromatic dispersion (CD) of the fiber. To overcome this limitation, several techniques can be used. One can operate near the zero dispersion region in the O-band but the increased losses can be limiting. If operation in C-band is considered, more dispersion tolerant modulation formats can be selected. One can use multi-level signaling (e.g. PAM4, with increased power penalty), discretemulti tone (heavily relying on DSP), optical duobinary modulation, single sideband (SSB) or vestigial sideband modulation or combined amplitude and phase shift (CAPS) codes [1] [2]. Besides choosing a more dispersion tolerant modulation format, the impact of $\mathrm{CD}$ can be compensated using optical and electronic techniques. Dispersion compensating fiber or fiber Bragg gratings are efficient but introduce extra losses, are costly and can be bulky. A recent optical equalization filter for $\mathrm{CD}$ is found in [3] using tunable lattice filters, suffering from increased insertion loss. Electronic equalization after square law direct detection has limited performance due to its nonlinear characteristic. Recently, Kramers-Kroning receivers are

M. Verplaetse, L. Breyne, J. Van Kerrebrouck, P. Ossieur and G. Torfs are with Ghent University - imec, IDLab, Department of Information Technology (e-mail: michiel.verplaetse@ugent.be).

M. Verplaetse has a PhD Fellowship of the Research Foundation - Flanders.

Copyright (c) 2019 IEEE. Personal use of this material is permitted. However, permission to use this material for any other purposes must be obtained from the IEEE by sending a request to pubs-permissions@ieee.org proposed as a more efficient technique (e.g. [4]), but these solutions rely again on DSP. Equalizing the signal in the electrical domain before modulating the light has been used intensively in the past decades as it has the possibility to be an adaptive solution and has better performance compared to compensation after detection. In past literature, the electronic precompensation is commonly performed in the digital domain using different kinds of optical modulators. An IQ modulator consisting of two parallel Mach-Zehnder modulators (MZMs) is used very often (e.g. [5] [6]) as it is the most straightforward technique to directly control the electrical field of the light. An alternative modulation choice which uses a single dual-drive MZM can be found in [7]. Less optimal modulating techniques such as using a directly modulated laser are also combined with DSP to successfully precompensate CD [8], [9].

The solutions using an IQ modulator with digital filtering provide linear compensation of $\mathrm{CD}$ for which the performance is mainly limited by the implemented filter lengths and the digital-to-analog converter (DAC) accuracy. However, the simplicity and power reduction promised when using DD is lost when DSP is used. For example, the state-of-the art high speed DACs in [10] (6-bit, 64 GS/s) consume around $620 \mathrm{~mW}$ each. Complexity, power consumption and latency increase even more if a full DSP architecture is considered, including demuxing (and capturing) of the input signals, clock generation and the digital processing. Even for a limited amount of chromatic dispersion to be compensated (i.e. short filter lengths), these steps are necessary. To avoid this overhead in the case of limited fiber span, we propose to use an analog (electrical) compensation filter instead of DSP. In this paper, an MZM based IQ-modulator (further addressed as IQ-MZM) will be used. To perform the filtering, an analog complex finite impulse response (FIR) filter IC with sufficient bandwidth to handle 28 Gbaud signals is used. Due to the design complexity of an analog FIR filter, bandwidth restrictions will limit the longest filter that can be realized and hence the CD compensation capability. Therefore, we introduce a variable delay between the in-phase and quadrature input of the IQ modulator. By combining both the analog FIR filter and delay, real-time transmission experiments are performed in the Cband using $28 \mathrm{~Gb} / \mathrm{s}$ NRZ/OOK up to a distance of $60 \mathrm{~km}$ standard single-mode fiber (SSMF) without any form of DSP.

\section{ChromatiC DISPERSION COMPENSATION PRINCIPLE}

To create a DD link with the possibility to precompensate the $\mathrm{CD}$, the system in Fig. 1 is proposed. The input waveform is shaped by two analog filters $H_{\mathrm{I}}$ and $H_{\mathrm{Q}}$ and then applied 


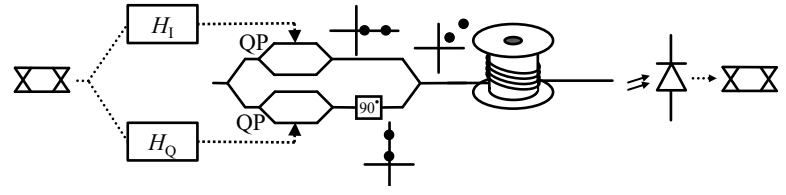

Fig. 1. Coherent precompensation system for direct detection systems suffering from chromatic dispersion.

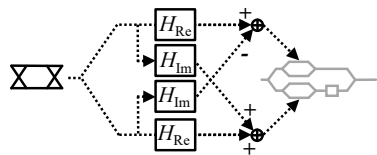

(a)

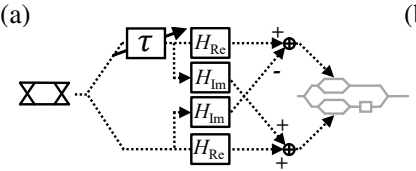

(c)

Fig. 2. Different representations of the precompensation filters from Fig. 1 (a) Showing the complex filter (butterfly representation), (b) shows the delay representation, and (c) shows the combined solution.

to a coherent modulator (IQ-MZM). The modulator has a $\pi / 2$ phase shift between both parallel MZMs biased at their quadrature points $(\mathrm{QP})$ and an applied swing that is not larger than the $\mathrm{V}_{\pi}$ of the MZMs. This shifts the transmitted constellation points into a single quadrant and allows DD at the receiver. If no $\mathrm{CD}$ is present in the link, the filter $H_{\mathrm{I}}$ and $H_{\mathrm{Q}}$ can be removed and both I and Q components of the field are modulated equally to obtain ASK modulation, which yields the correct output when direct detection is used. If dispersion is present, the filters $H_{\mathrm{I}}$ and $H_{\mathrm{Q}}$ are needed to compensate the $\mathrm{CD}$. A first possible representation for both filters is found in Fig. 2a. Here they implement a complex compensation filter $H_{c}=H_{\mathrm{Re}}+j H_{\mathrm{Im}}$. The filters $H_{\mathrm{I}}$ and $H_{\mathrm{Q}}$ can respectively be implemented as $H_{\mathrm{Re}}-H_{\mathrm{Im}}$ and $H_{\mathrm{Re}}+H_{\mathrm{Im}}$. The filter $H_{c}$ should be shaped as close as possible to the inverse of the complex valued $\mathrm{CD}$ fiber response. For the implementation, FIR filters can be used either in the digital (DSP) or the analog domain. The filter coefficients for a certain amount of CD, a given number of filter taps and their spacing can be optimized numerically. The optimization, performed in simulation, minimizes the inter-symbol-interference in the electric field incident on the photoreceiver. Simulations show that for the compensation of $28 \mathrm{~Gb} / \mathrm{s} \mathrm{NRZ}$ transmission over $50 \mathrm{~km}$ of SSMF with limited penalty, 13 half symbol spaced FIR taps or more are necessary. While this is still a low number of FIR taps for a digital filter, this number of taps would already be higher than state-of-the-art reported $\geq 25$ Gbaud analog FIR-equalizer designs (e.g. 7 taps in [11], 6 taps in [12]). An analog FIR-equalizer with 5 half symbol-spaced taps, can compensate around $15 \mathrm{~km}$ of fiber with limited penalty. To extend the reach to $20 \mathrm{~km}$ with 5 analog taps, the tap spacing could be increased slightly towards $0.75 \mathrm{~T}_{\mathrm{s}}$ (with $\mathrm{T}_{\mathrm{s}}$ the symbol period), creating a longer filter response without implications on the link performance [11]. The optimal filter parameters in this case for $20 \mathrm{~km}$ of fiber are given in Fig. 3 together with the respective frequency responses of the real and imaginary parts of the filter. (a)

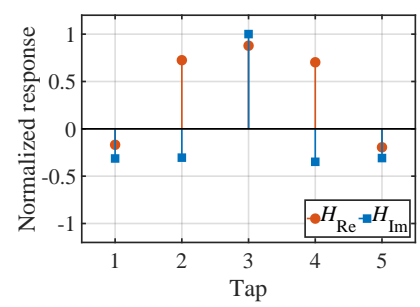

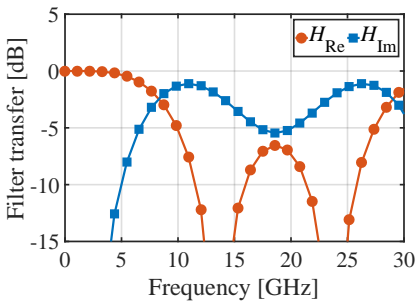

(b)
Fig. 3. (a) Optimized filter parameters in simulation for $20 \mathrm{~km}$ of fiber with 5 analog taps and $0.75 T_{\mathrm{s}}$ spacing. (b) Corresponding frequency responses.

Alternatively to shaping the filter towards the inverse of the $\mathrm{CD}$ response, one can replace either $H_{\mathrm{I}}$ or $H_{\mathrm{Q}}$ with a broadband delay and the other with a thru connection, as shown in Fig. 2b. This alternative filter approach creates optical SSB modulation in a limited part of the signal spectrum depending on the implemented delay value: for the electrical frequency for which the delay equals a $\pi / 2$ electrical phase shift, the resulting structure is a single-sideband modulator. Simulations show that the interplay of this precompensated signal with the complex CD response opens the eye diagram up to $20 \mathrm{~km}$ of SSMF, while it is completely closed without delay between the I and the $\mathrm{Q}$ input.

Both methods can be combined as in Fig. 2c, which is possible as the butterfly filter creates virtually a shorter fiber span without changing the interface towards the input waveform. In this way the fiber reach can be increased when only a limited number of analog FIR taps are available by adding a single variable delay component.

\section{EXPERIMENTAL SETUP}

The experimental setup used to verify the proposed compensation techniques is shown in Fig. 4. To generate the necessary filters, an in-house designed analog complex equalizer IC is used. The chip implements four real valued filters in a butterfly structure (see inset Fig. 4c). Each filter is an independently tunable five tap FIR filter with a tap spacing of $\approx 27$ ps $\left(\approx 0.75 \mathrm{~T}_{\mathrm{s}}\right.$ at $\left.28 \mathrm{~Gb} / \mathrm{s}\right)$ with a maximum small-signal gain up to $6 \mathrm{~dB}$ for each single tap. The chip is designed in a $55 \mathrm{~nm}$ BiCMOS technology and consumes $340 \mathrm{~mW}$ (using all four FIR filters and including high-speed input/output stages) from a $2.5 \mathrm{~V}$ supply. In theory, only 2 out of the 4 filters are necessary to implement the proposed concept from Fig. 1 with the different representations in Fig. 2. If all four filters are used, the concept in Fig. 2c can be directly tested by adding an external variable delay to one of the 2 inputs of the chip (also shown in Fig. 4). This approach will ease verification as the filters used to test the direct $\mathrm{CD}$ compensation in Fig. 2a can be reused for the concept in Fig. 2c. Secondly, performance can also be slightly better as the delay does not has to be absorbed in the analog filters, which would lower the useful filter length. As the single ended output swing of the equalizer chip is set to be $\approx 150 \mathrm{mVpp}$, two broadband $\mathrm{RF}$ amplifiers with $24 \mathrm{~dB}$ gain are used to drive the IQMZM. The IQ-MZM is part of a commercial dual polarization coherent transmitter (LiNbO3 MZM - Fujitsu FTM7992HM) 


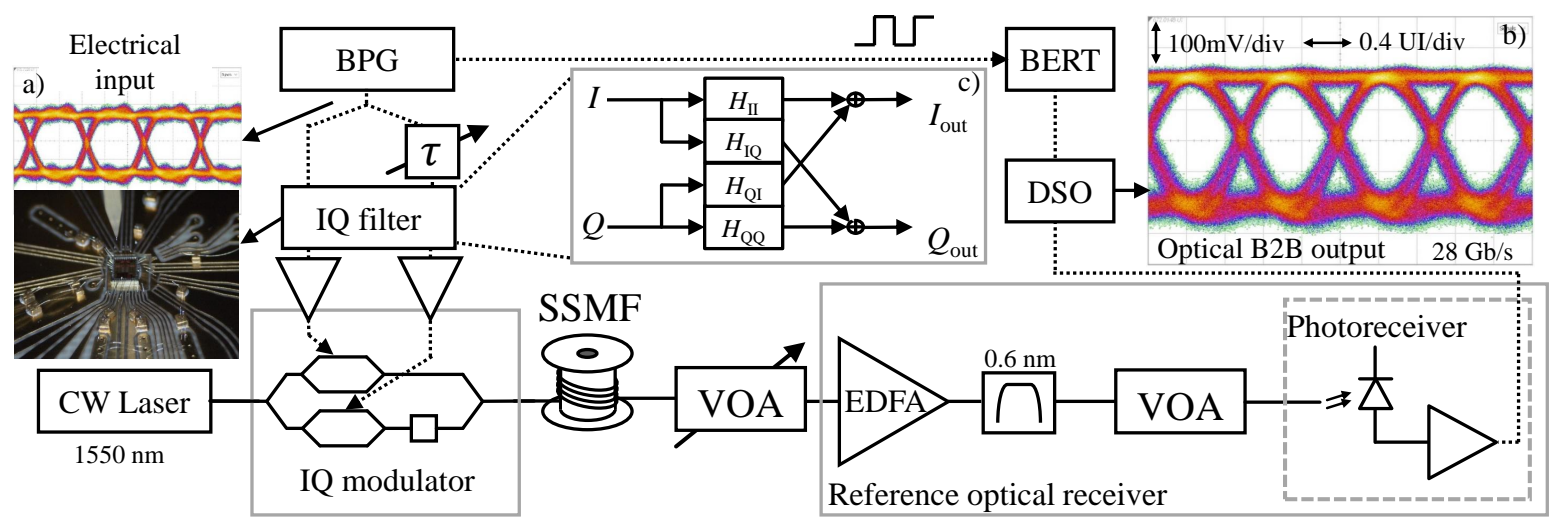

Fig. 4. Overview of the setup used in the experiment. (a) Shows the electrical input eye diagram at $28 \mathrm{~Gb} / \mathrm{s}$ provided to the IQ filter. (b) Gives the eye diagram at $28 \mathrm{~Gb} / \mathrm{s}$ at the output of the photoreceiver in the optical B2B case. (c) Block diagram of the different analog filters in the IQ filter chip.

with a maximum $\mathrm{V}_{\pi}$ of $4.2 \mathrm{~V}$. The IQ-MZM of the $\mathrm{y}$ polarization is unused and biased at its minimum transmission point. Both MZMs in the x-polarization are biased at their quadrature point as discussed before. The $\mathrm{CW}$ laser launches $13 \mathrm{dBm}$ into the modulator at a wavelength of $1550 \mathrm{~nm}$. The modulator has $18.1 \mathrm{~dB}$ loss, resulting in $-5.1 \mathrm{dBm}$ power launched into the fiber $(\approx 1 \mathrm{dBm}$ of power is expected if a dedicated single IQ-MZM were used). The modulated signal is then sent through different lengths of SSMF. At the fiber output, a variable optical attenuator (VOA) is added to control the optical power incident on the receiver. In the receiver, the signal is optically preamplified with an EDFA and filtered with a tunable bandpass filter, centered around $1550 \mathrm{~nm}$ with a total bandwidth (FWHM) of $0.6 \mathrm{~nm}$. A second VOA is used to limit the incident power on the photoreceiver to $0 \mathrm{dBm}$. This receiver is a commercial linear PIN differential photoreceiver (DSC-R409-LW from Discovery Semiconductors, Inc.) with a bandwidth of $31 \mathrm{GHz}$ and a conversion gain of $318 \mathrm{~V} / \mathrm{W}$. As shown in Fig. 4, the EDFA and photoreceiver form a reference optical receiver with an increased sensitivity to characterize the precompensation system at long fiber spans. The receivers output signal is sent either to a high-speed sampling scope (DSO) to observe the eye diagrams or to a BER tester for real time BER measurements (using the transmitter clock). Throughout the remainder of this paper a $28 \mathrm{~Gb} / \mathrm{s}$ NRZ/OOK pseudo random bit sequence (PRBS) test pattern with a length of $2^{9}-1$ will be used.

\section{RESULTS AND DISCUSSION}

To evaluate the performance of the discussed compensation principle, different experiments were conducted using the setup in Fig. 4. The BER was measured for different fiber reaches as a function of the optical power at the input of the EDFA. The power was swept by varying the attenuation of the VOA at the input. First a reference measurement was done where no IQ filtering or delay is applied to the input signals. In this measurement, the equalizer chip was configured such that $H_{\mathrm{IQ}}\left(H_{\mathrm{QI}}\right)$ is zero and $H_{\mathrm{II}}\left(H_{\mathrm{QQ}}\right)$ acts as a thru. The observed eye diagram for the back-to-back (B2B) case is found in Fig. 4b. From the B2B eye diagram and the responsivity of the photoreceiver, an extinction around $6.4 \mathrm{~dB}$ is found. After $20 \mathrm{~km}$ of fiber, the eye diagram was almost closed which is expected as the spectral notch, caused by the $\mathrm{CD}$ at this distance is very close to the Nyquist frequency of $14 \mathrm{GHz}$. The measured BERs for different fiber lengths, together with the annotation of two commonly used FEC limits (7\%-OH $\left.\mathrm{HD}-\mathrm{FEC}=3.8 \times 10^{-3}, \mathrm{KP}-4 \mathrm{FEC}=2.4 \times 10^{-4}\right) \mathrm{can}$ be found in Fig. 5-(a). In a second experiment, while keeping the analog filter response transparent, the delay is optimized to reach a minimal BER at different transmission distances. The BER curves for these experiments are found in Fig. 5-(b) showing an increased reach up to $30 \mathrm{~km}$ below the KP4-FEC. The applied delays between the I and Q signals for the $10 \mathrm{~km}$, $20 \mathrm{~km}$ and $30 \mathrm{~km}$ fiber spans are respectively $6 \mathrm{ps}, 35 \mathrm{ps}$ and 39 ps. In a third experiment, the IQ filter is set with the complex filter parameters (determined by simulation as discussed in section II, resimulated for each fiber span) to compensate the $\mathrm{CD}$ in the fiber while no delay between the I and Q inputs is applied. It should be noted that no further optimization on the tap coefficients was performed, only the total gain of the filter was tuned to avoid possible clipping at the outputs of the chip. The BER curves for these experiments are found in Fig. 5-(c). Error free operation is obtained up to $20 \mathrm{~km}$ as expected by simulation. Transmission over larger distances is also possible with an increased power penalty (e.g. $7 \mathrm{~dB}$ vs. $\mathrm{B} 2 \mathrm{~B}$ for $30 \mathrm{~km}$ at a $\mathrm{BER}=2.4 \times 10^{-4}$ ). As a fourth experiment, the complex filter parameters needed for the linear compensation of $20 \mathrm{~km} \mathrm{SSMF}$ are loaded into the IQ filter and the delay $\tau$ in Fig. 4 is optimized to minimize the BER. In this way, transmission over $60 \mathrm{~km}$ of SSMF with a BER of $3.7 \times 10^{-3}$ (below the 7\%-OH-HD FEC) is obtained using a delay of 31 ps. For all shorter distances, BERs below the KP4FEC limit are achieved, as shown in Fig. 5-(d). Different output eye diagrams of this fourth experiment are found in Fig. 6. If the EDFA and VOAs are removed, transmission over $20 \mathrm{~km}$ and $30 \mathrm{~km}$ of SSMF is demonstrated with respectively BERs of $8.4 \times 10^{-7}$ and $2.57 \times 10^{-3}$ mainly limited by the sensitivity of the photoreceiver as the average optical received power is respectively -10.16 and $-13.1 \mathrm{dBm}$. In Fig. 7 , the minimum power needed to reach a BER of $3.8 \times 10^{-3}$ for the different experiments is summarized. For a power penalty (versus the B2B case) of $3 \mathrm{~dB}$, no filtering results in approximately $13 \mathrm{~km}$ reach, which increases to $20 \mathrm{~km}$ in the case of the delay and $\approx 25 \mathrm{~km}$ in the other two cases. For a minimal power of 


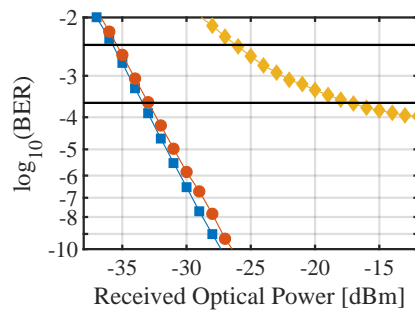

(a)

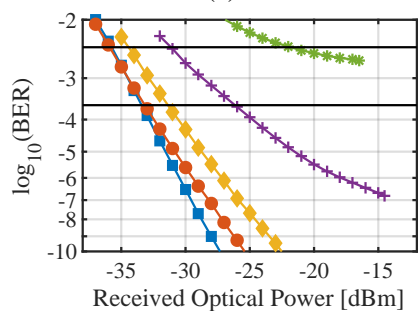

(c)

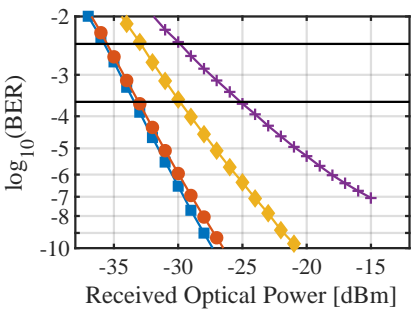

(b)

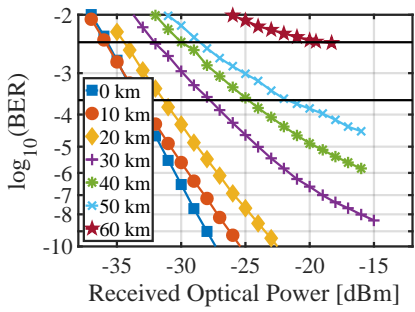

(d)
Fig. 5. BER curves in function of the received optical power for (a) no compensation, (b) only delay compensation (c) only IQ filtering (d) combined delay and IQ filtering.
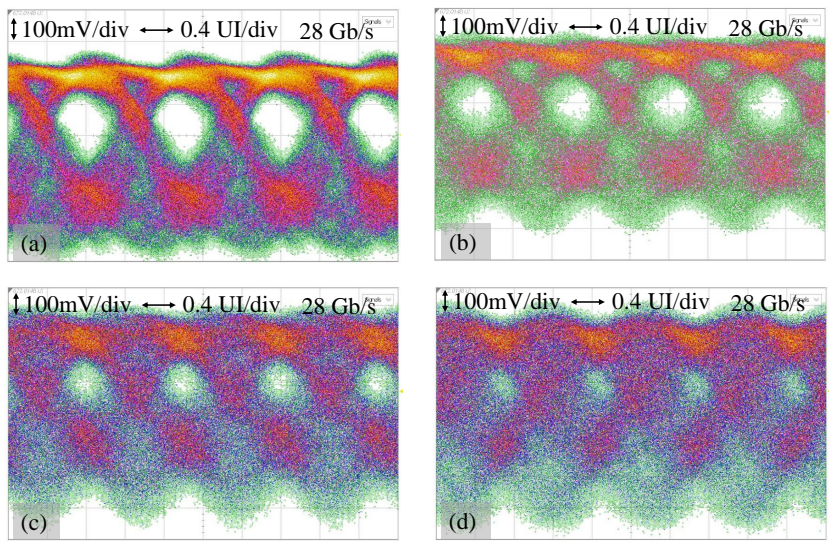

Fig. 6. Different eye diagrams at the receiver using both IQ filtering and delay compensation for (a) 20, (b) 30, (c) 50 and (d) $60 \mathrm{~km}$ of SSMF.

$-22 \mathrm{dBm}$, the reach increases further from $\approx 40 \mathrm{~km}$ with only IQ filtering to $\approx 56 \mathrm{~km}$ using both IQ filtering and delay. This shows the increased range extension that can be obtained by combining both methods which eventually leads to the reach of $60 \mathrm{~km}$ at a minimum required power of $-19 \mathrm{dBm}$.

\section{CONCLUSION}

We demonstrate an analog precompensation technique which allows to significantly improve the chromatic dispersion tolerance in direct detection links. The analog precompensation is proposed to be a low complexity, low power consumption and lower latency alternative compared to DSP based compensation if only a limited amount of chromatic dispersion is present in the fiber. The technique uses an IQ modulator and an in-house designed complex analog 5-tap FIR equalization chip to linearly precompensate the chromatic dispersion at the transmit side. The fiber reach is further increased by introducing a tunable delay between the I and $\mathrm{Q}$ signal of the IQ modulator. The combination results in a

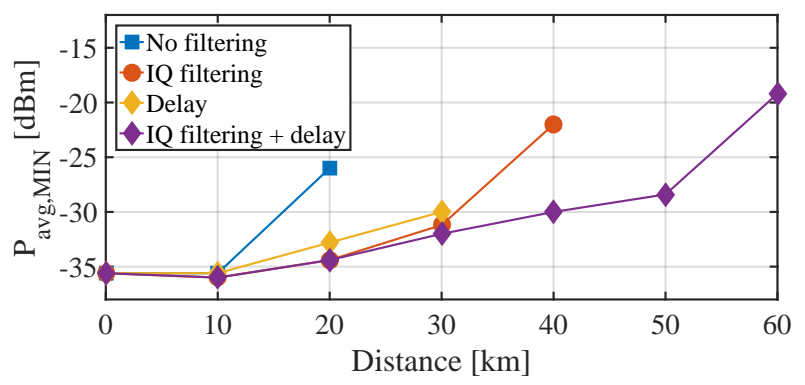

Fig. 7. The minimal received optical power for a BER below the $7 \%-\mathrm{OH}$ HD FEC limit for the different CD compensations methods.

real-time transmission experiment of $28 \mathrm{~Gb} / \mathrm{s} \mathrm{NRZ/OOK}$ in the C-band over $60 \mathrm{~km}$ of SSMF with a BER below the $7 \%-\mathrm{OH}$ HD-FEC without any digital signal processing or dispersion compensating fiber. Additionally, real-time transmission over $30 \mathrm{~km}$ is demonstrated below the $7 \%-\mathrm{OH}$ HD-FEC limit $\left(3.8 \times 10^{-3}\right)$ without optical pre-amplification.

\section{ACKNOWLEDGMENT}

We acknowledge the ERC Advanced Grant 695495 (ATTO), the Special Research Fund and the Research Foundation Flanders for funding and VPIphotonics for its software.

\section{REFERENCES}

[1] M. Morsy-Osman et al., "50 Gb/s Short-reach interconnects with DSPfree direct-detection enabled by CAPS codes," Opt. Express, vol. 26, no. 14, pp. 17916-17926, Jul 2018.

[2] M. Chagnon, "Optical communications for short reach," Journal of Lightwave Technology, vol. 37, no. 8, pp. 1779-1797, April 2019.

[3] G. Brodnik et al., "Extended reach $40 \mathrm{~km}$ transmission of C-band realtime 53.125 Gbps PAM-4 enabled with a photonic integrated tunable lattice filter dispersion compensator," in Optical Fiber Communications Conference, March 2018, pp. 1-3.

[4] Z. Li et al., "SSBI mitigation and the Kramers-Kronig scheme in singlesideband direct-detection transmission with receiver-based electronic dispersion compensation," Journal of Lightwave Technology, vol. 35, no. 10, pp. 1887-1893, May 2017.

[5] J. McNicol et al., "Electrical domain compensation of optical dispersion," in Optical Fiber Communication Conference, vol. 4, March 2005.

[6] Z. Liu et al., "448-Gb/s PAM4 transmission over 300-km SMF-28 without dispersion compensation fiber," in Optical Fiber Communications Conference, March 2018, pp. 1-3.

[7] R. I. Killey et al., "Electronic dispersion compensation by signal predistortion using digital processing and a dual-drive Mach-Zehnder modulator," IEEE Photonics Technology Letters, vol. 17, no. 3, pp. 714716, March 2005.

[8] S. Warm et al., "Electronic dispersion precompensation with a $10-\mathrm{Gb} / \mathrm{s}$ directly modulated laser," IEEE Photonics Technology Letters, vol. 21, no. 15, pp. 1090-1092, Aug 2009.

[9] A. S. Karar, J. C. Cartledge, and K. Roberts, "Transmission over 608 km of standard single-mode fiber using a $10.709-\mathrm{Gb} / \mathrm{s}$ chirp managed laser and electronic dispersion precompensation," IEEE Photonics Technology Letters, vol. 24, no. 9, pp. 760-762, May 2012.

[10] J. Cao et al., "A transmitter and receiver for $100 \mathrm{~Gb} / \mathrm{s}$ coherent networks with integrated 4x64GS/s 8b ADCs and DACs in 20nm CMOS," in 2017 IEEE International Solid-State Circuits Conference (ISSCC), Feb 2017, pp. 484-485.

[11] E. Mammei et al., "Analysis and design of a power-scalable continuoustime FIR equalizer for $10 \mathrm{~Gb} / \mathrm{s}$ to $25 \mathrm{~Gb} / \mathrm{s}$ multi-mode fiber EDC in 28 nm LP CMOS," IEEE Journal of Solid-State Circuits, vol. 49, no. 12, pp. 3130-3140, Dec 2014.

[12] J. Verbist et al., "Real-Time $100 \mathrm{~Gb} / \mathrm{s}$ NRZ and EDB Transmission With a GeSi Electroabsorption Modulator for Short-Reach Optical Interconnects," Journal of Lightwave Technology, vol. 36, no. 1, pp. 90-96, Jan 2018. 